\title{
Dynamic and kinematic observers for output coordination control of Euler-Lagrange systems: A comparison and applications
}

\author{
E. Kyrkjebø \\ Sogn and Fjordane University College, N-6851 Sogndal, Norway.E-mail: Erik.Kyrkjebo@hisf.no
}

\begin{abstract}
This paper compares a dynamic and a kinematic observer approach for output coordination control of mechanical systems formulated in the Euler-Lagrange framework. The observers are designed to estimate missing velocity and acceleration information based on position/attitude measurements to provide a full state vector to the coordination control algorithm. The kinematic observer approach utilizes a virtual system designed to mimic the kinematic behaviour of the leader in order to estimate unknown states of the state vector with a minimum of information available. The dynamic observer approach is based on utilizing the full dynamic model of the follower system when estimating the missing states. The two observers are compared in terms of estimation principles and practical performance, and applied to two practical examples; leader-follower robot manipulator synchronization control, and underway replenishment operations for surface ships.
\end{abstract}

Keywords: Observer, Synchronization, Coordination, Control, Surface ship, Robot

\section{Introduction}

The output coordination control problem - where the control objective of a follower is to coordinate its motion to a leader for which only position/attitude measurements are available - is found in a wide range of applications for mechanical systems; robot manipulators, mobile robots, ships, aeroplanes and multi-satellite systems. The coordination problem is important when two or more systems must operate in time with each other, and where simple single-system control approaches lack the necessary synchronization mechanisms to ensure timely operation between them.

Coordinating the motion of two or more systems requires that some information must be available on the states of the systems, and in many applications only position/attitude information of the external leader system can be expected to be readily available as measurements. Robot manipulators are often equipped with high-precision position sensors such as encoders, but velocity or acceleration measurements are not so readily available, or are contaminated with noise when obtained from low-quality tachometers. Some sort of estimation technique must therefore be designed to provide good estimates of the missing states. However, it may be difficult to obtain full knowledge of mathematical models or commanded control signals of external systems to design model-based observers of the external states. In addition, simple differentiation techniques to obtain velocity and acceleration estimates from measurements are often noisy and prone to contamination by wild-points. Therefore, the observer design problem for output coordination control must be carefully addressed to provide valid velocity and acceleration estimates to the control algorithms.

This paper compares a kinematic and a dynamic observer approach to the output coordination control problem in terms of estimation principle and performance in the presence of disturbances and model errors. The main tradeoffs between the two approaches to provide guidelines for choosing the appropriate observer design for a particular coordination control application will also be discussed.

Luenberger (1971) suggests that "Almost any System is 
an Observer". If the available outputs of a system are used as inputs to drive another system, the second system will almost always serve as an observer of the first system in that its states will tend to track the states of the first system. This feature has been extensively exploited in control theory over the years, and observers provides estimates of unknown or uncertain states in many practical implementations where the measurements are not available directly, are contaminated by noise or are prone to measurement dropouts. However, the observer principles utilized in these implementations range from simple differentiation techniques to estimate velocity from position measurements, to non-linear observers utilizing the full dynamic model of the system to estimate all unmeasured states of a system. In the following, the estimation principle of two such observers and how it will influence the performance of a system as evidenced by robustness towards external disturbances, measurement noise, actuator limitations and modelling errors is investigated. A simple kinematic observer based on the principle of a virtual system is compared with a more complex full-order non-linear dynamic model-based observer in an output coordination control problem where only position/attitude measurements are available for the external leader system.

The objective of an output coordination control scheme is to synchronize the motion of a follower to a leader - in effect the follower should become a physical observer of the leader following the statement of Luenberger (1971). The observer design problem is further complicated due to the fact that the parameters of the mathematical model of the leader are considered unknown (facilitating a wider range of motion coordination applications, and also applications where the leader may not be a mechanical system, i.e. a human operator). The dynamic model of the leader may thus not be used directly when estimating the unknown states of that system. An overview of observers for non-linear systems can be found in Marino and Tomei (1995) and Nijmeijer and Fossen (1999), while observers for synchronization control are discussed in Nijmeijer and Mareels (1997) and Pogromsky and Nijmeijer (1998). Recent results on output synchronization control of robots manipulator can be found in Nishigami et al. (2009), for mobile robots in Choi et al. (2010) and for marine vessels in Peng et al. (2013).

The kinematic observer approach utilizes a virtual system as an intermediate system between the leader and the follower. The virtual system approach has been utilized both as an abstraction vehicle in Crowley (1989), and as an intermediate level between the desired trajectories of a system and the controller. The virtual system can be considered as a low-level controller in a two-level control structure (Fradkov et al., 1991; Gusev et al., 1998). A kinematic observer that reconstructs the velocity of the leader for adaptive formation control has also been designed in Choi et al. (2010). The kinematic observer approach in this paper follows the design in Kyrkjebø et al. (2006a) where a virtual system follows the behaviour of the leader based on position/attitude feedback only. The leader is a dynamic system manoeuvring freely, and the parameters of the mathematical model, the control inputs and internal states of the leader are unknown to the follower. The virtual system is designed to stabilize its trajectories to the position of the leader through a kinematic control law, and the coordination control law of the follower is provided with an estimate of the states of the leader through this definition of the virtual control law. The virtual system approach imposes a cascaded structure in the control system through a separation of the observer and controller design, as opposed to the dynamic modelbased approach where the observer and controller are more closely interconnected.

The dynamic model-based observer approach has been widely applied in control problems for mechanical systems; particularly to tracking control for general mechanical systems (Loría et al., 1997; Lefeber, 2000), mobile robots (Salichs et al., 1991), marine systems (Pettersen and Nijmeijer, 1998; Encarnacao and Pascoal, 2001), flightcontrol (Al-Hiddabi and McClamroch, 2002), and many others. These output tracking control approaches assume that state information of the reference trajectory is available, but Rodriguez-Angeles and Nijmeijer (2001) presented an output coordination control approach for two robot manipulators based on only position measurements of the leader. This synchronization approach facilitates 'tracking" control of systems where the states of the reference trajectory are unknown, and was later utilized in Nijmeijer and Rodriguez-Angeles (2003) for both coordination and cooperative control of mechanical systems.

The dynamic observer approach follows the design in Kyrkjeb $\varnothing$ and Pettersen (2007) where a model-based observer estimates the unknown states of a leader based on only position/attitude measurements of the leader. The dynamic observer design is based on utilizing information about the model and control signals of the follower to filter the closed-loop errors of the coordination control scheme to generate estimates of the derivatives of the closed-loop errors. When the states of the follower are known together with these error estimates, the leader states can be estimated through algebraic manipulation. The control input to the follower from the coordination control law is based on these estimates of the leader, and can be thought of as part of the correction term in the dynamic observer.

The main contribution of this paper is the comparison of estimation principles and performance of a kinematic and a dynamic observer scheme for output coordination control. A shorter version of the results has been presented in Kyrkjeb $\varnothing$ and Pettersen (2009). The comparison between the kinematic (virtual system) observer approach (based on Kyrkjebø et al. (2006a)) and the dynamic (model-based) observer approach (based on Kyrkjebø and Pettersen (2007)) 
is qualitative - it addresses the difference in estimation principles and the inherent response to disturbances rather than quantifying performance results. The two observer approaches react differently to different types of disturbances, and this paper focuses on providing knowledge and guidelines for choosing estimation principles for a wide range of coordination applications through a comparison of the two presented observer schemes. The two observers are also discussed in the context of two practical applications of output coordination control; synchronization of two robot manipulators (based on Kyrkjebø and Pettersen (2008)) and underway replenishment operations for surface ships (based on Kyrkjebø et al. (2006b)).

This paper is organized as follows; Section 2 presents the kinematic and dynamic observer principles, Section 3 applies the two observers in two coordination control examples, Section 4 discusses the performance of the two observer schemes, and concluding remarks are presented in Section 5.

\section{Estimation principles}

For completeness, a brief presentation of the kinematic and dynamic model-based observer schemes that will be compared in Section 4 is given. More details of the schemes can be found in Kyrkjeb $\varnothing$ et al. (2006a) and Kyrkjeb $\varnothing$ and Pettersen (2007). The two observer designs are utilized in the output leader-follower coordination control problem where a fully actuated follower, for which all states are measured and all mathematical parameters are known, synchronizes its motion to the motion of a leader. The parameters of the mathematical model and control signals of the leader are considered unknown, and the observer schemes estimate the states of the leader based on position/attitude measurements only.

\subsection{Preliminaries}

The two observer schemes presented are valid for the output leader-follower coordination control problem of mechanical systems described by the Euler-Lagrange equations (Goldstein et al. (2002))

$$
\frac{d}{d t}\left(\frac{\partial \mathscr{L}(\mathbf{x}, \dot{\mathbf{x}})}{\partial \dot{\mathbf{x}}}\right)-\frac{\partial \mathscr{L}(\mathbf{x}, \dot{\mathbf{x}})}{\partial \mathbf{x}}+\frac{\partial \mathscr{F}(\dot{\mathbf{x}})}{\partial \dot{\mathbf{x}}}=\tau
$$

where $\mathbf{x} \in \mathbb{R}^{n}$ are generalized coordinates assumed measurable, and $\tau \in \mathbb{R}^{n}$ are generalized forces acting on the system. $\mathscr{L}(\mathbf{x}, \dot{\mathbf{x}})=\mathscr{T}(\mathbf{x}, \dot{\mathbf{x}})-\mathscr{V}(\mathbf{x})$ is the Lagrangian function of potential energy $\mathscr{V}(\mathbf{x})$ and kinetic energy $\mathscr{T}(\mathbf{x}, \dot{\mathbf{x}})$. It is assumed that the kinetic energy function is of the quadratic form

$$
\mathscr{T}(\mathbf{x}, \dot{\mathbf{x}})=\frac{1}{2} \dot{\mathbf{x}}^{T} \mathbf{M}(\mathbf{x}) \dot{\mathbf{x}}, \quad \mathbf{M}(\mathbf{x})=\mathbf{M}^{T}(\mathbf{x})>0
$$

where the inertia matrix $\mathbf{M}(\mathbf{x})$ is positive definite and uniformly bounded. Using the Christoffel symbols of the first kind from Spong and Vidyasagar (1989) and (2), (1) can be rewritten in the form of an Euler-Lagrange system with dynamics as

$$
\mathbf{M}(\mathbf{x}) \ddot{\mathbf{x}}+\mathbf{C}(\mathbf{x}, \dot{\mathbf{x}}) \dot{\mathbf{x}}+\mathbf{d}(\mathbf{x}, \dot{\mathbf{x}})+\mathbf{g}(\mathbf{x})=\tau
$$

where $\mathbf{C}(\mathbf{x}, \dot{\mathbf{x}}) \dot{\mathbf{x}}$ is the vector of Coriolis and centripetal forces, and the vector of potential forces is given by $\mathbf{g}(\mathbf{x})$. The model (3) is an Euler-Lagrange system (Kyrkjeb $\varnothing$ and Pettersen, 2005), and satisfies the following properties (Ortega and Spong, 1989)

P1 $\mathbf{M}(\mathbf{x})$ satisfy $0<\mathbf{M}_{m} \leq\|\mathbf{M}(\mathbf{x})\| \leq \mathbf{M}_{M}<\infty$, where $\mathbf{M}_{m}$ and $\mathbf{M}_{M}$ are positive constants.

P2 $\mathbf{M}(\mathbf{x})$ is differentiable in $\mathbf{x}$ and $\mathbf{y}^{T}(\dot{\mathbf{M}}(\mathbf{x})-2 \mathbf{C}(\mathbf{x}, \dot{\mathbf{x}})) \mathbf{y}=0, \forall \mathbf{x}, \mathbf{y} \in \mathbb{R}^{n}$.

P3 $\mathbf{C}(\mathbf{x}, \mathbf{y}) \mathbf{z}=\mathbf{C}(\mathbf{x}, \mathbf{z}) \mathbf{y}$, and also $\|\mathbf{C}(\mathbf{x}, \dot{\mathbf{x}})\| \leq \mathbf{C}_{M}\|\dot{\mathbf{x}}\|$.

The dissipative or frictional forces in the system are derived from the scalar dissipation function $\mathscr{F}(\dot{\mathbf{x}})$, defined from the rate of energy $\mathscr{E}=-\mathscr{F}(\dot{\mathbf{x}})$ dissipating from the system as

$$
\mathbf{d}(\mathbf{x}, \dot{\mathbf{x}})=\mathbf{D}(\mathbf{x}, \dot{\mathbf{x}}) \dot{\mathbf{x}}=\frac{\partial \mathscr{F}(\dot{\mathbf{x}})}{\partial \dot{\mathbf{x}}}
$$

Assumption 1 The dissipative term $\mathbf{d}(\mathbf{x}, \dot{\mathbf{x}})$ is continuously differentiable in $\mathbf{x}$ and $\dot{\mathbf{x}}$, and satisfies for some $k_{d} \geq 0$

$$
\mathbf{y}^{T} \frac{\partial \mathbf{d}(\mathbf{x}, \dot{\mathbf{x}})}{\partial \dot{\mathbf{x}}} \mathbf{y} \geq k_{d} \mathbf{y}^{T} \mathbf{y}, \quad \forall \mathbf{x}, \dot{\mathbf{x}}, \mathbf{y} \in \mathbb{R}^{n}
$$

and for a continuous function $\beta_{d}(\mathbf{s}): \mathbb{R}_{\geq 0} \rightarrow \mathbb{R}_{\geq 0}$

$$
\left\|\frac{\partial \mathbf{d}(\mathbf{x}, \dot{\mathbf{x}})}{\partial \dot{\mathbf{x}}}\right\| \leq \beta_{d}(\|\dot{\mathbf{x}}\|), \quad \forall \mathbf{x}, \dot{\mathbf{x}} \in \mathbb{R}^{n}
$$

Note that Assumption 1 is a generalization based on practical considerations regarding the dissipative terms in most Euler-Lagrange systems, and that the presence of dissipative forces are mainly due to friction or hydro- and aerodynamic damping effects. In particular, the restrictiveness of Assumption 1 is addressed for friction effects in Section 3.1, and for hydrodynamic damping effects in Section 3.2.

A differential kinematic relationship relates the bodyfixed velocities $v$ to a set of inertial generalized velocities $\dot{\mathbf{x}}$ through a Jacobian transformation matrix as

$$
\dot{\mathbf{x}}=\mathbf{J}(\mathbf{x}) v
$$

The estimation principles of the kinematic and dynamic model-based observer scheme are based on the notion of estimating the unknown states of the leader through a system that mimics (or simulates) the behaviour of the leader. 
In the kinematic observer scheme, this system is a virtual system that is constructed to stabilize to the output of the leader, and which in turn provides estimates of the states of the leader to the follower. For the dynamic model-based observer scheme, the mimicking system is the follower itself, and through the observers and controller the follower becomes a physical observer of the leader.

Information constraints are imposed on the proposed coordination schemes by allowing the parameters of the mathematical model of the leader to be unknown, and also by the fact that only the position is available as output from the leader, This suggest that the coordination control scheme with the two observers will not make the closed-loop errors converge to an equilibrium point at the origin, but rather to a bounded or practically stable solution about the origin. In particular, the presence of non-vanishing perturbations due to the unknown states render the closed-loop errors of the coordination control schemes at best ultimately bounded or practically stable, depending on the choice of observer.

\subsection{A kinematic observer approach}

The kinematic observer approach (Kyrkjeb $\varnothing$ et al., 2006a) is based on using a virtual (sub)copy of the system to act as an estimator for the unknown states of the leader through a virtual control law. This approach utilises the fact that while the full dynamic model of the leader is not readily computed, the kinematic model of the leader is relatively easy to obtain, and thus can more readily form the basis of the virtual system. In order to make the states of this kinematic model estimate the motion of the leader, a virtual control law is defined. This is designed by backstepping methods to stabilize the trajectories of the virtual system to the position of the leader. The virtual system thus provides an estimate of the position and velocity of the leader, which can be used as inputs to the coordination control law of the follower. The virtual system approach imposes a cascaded structure in the control system that can be exploited in the design and stability analysis.

The only measurement available from the leader is the position/attitude measurements $\mathbf{x}_{m}$ (subscript $m$ designates the leader system, sometimes referred to as master). No information of the parameters of the mathematical model or the control signals of the leader is assumed. Thus, the virtual system is designed as an intermediate controlled system stabilizing to the leader based only on position measurements. Consequently, the virtual system will provide estimates of the unmeasured states of the leader. As in Gusev et al. (1998), the first step (kinematic level) considers the velocities $v_{v}$ of the virtual system as the control inputs, and the control law is designed such that convergence of the virtual trajectories to the leader trajectories is ensured. In a way, the trajectories $\mathbf{x}_{v}$ and velocities $v_{v}$ can be considered as estimates of the leader states $\mathbf{x}_{m}$ and $v_{m}$. Thus, the virtual system becomes a kinematic estimator of the leader states through the position feedback loop. The virtual system can be defined by its kinematic model from (7) as

$$
\dot{\mathbf{x}}_{v}=\mathbf{J}\left(\mathbf{x}_{v}\right) v_{v}
$$

The kinematics of the leader is given by

$$
\dot{\mathbf{x}}_{m}=\mathbf{J}\left(\mathbf{x}_{m}\right) v_{m}
$$

The tracking errors of the virtual system are defined as

$$
\mathbf{e}_{v}=\mathbf{x}_{v}-\mathbf{x}_{m}, \quad \dot{\mathbf{e}}_{v}=\dot{\mathbf{x}}_{v}-\dot{\mathbf{x}}_{m}=\mathbf{J}\left(\mathbf{x}_{v}\right) v_{v}-\mathbf{J}\left(\mathbf{x}_{m}\right) v_{m}
$$

To stabilize the virtual system to the leader, a virtual system control law is proposed

$$
v_{v}=-\mathbf{J}^{-1}\left(\mathbf{x}_{v}\right) \mathbf{L}_{1} \mathbf{e}_{v}-\mathbf{J}^{-1}\left(\mathbf{x}_{v}\right) \mathbf{L}_{2} \mathbf{z}
$$

where $\mathbf{L}_{1}$ and $\mathbf{L}_{2}$ are symmetric positive gain matrices, and where the term $\dot{\mathbf{z}}=\mathbf{e}_{v}$ is added to introduce an integral term. The closed-loop error equation thus becomes

$$
\dot{\mathbf{e}}_{v}=-\mathbf{L}_{1} \mathbf{e}_{v}-\mathbf{L}_{2} \mathbf{z}-\mathbf{J}\left(\mathbf{x}_{m}\right) v_{m}
$$

Theorem 1 The closed-loop error dynamics (12) of system (8) where $\sup _{t}\left\|v_{m}\right\|=V_{M}<\infty$ with the control law (11) is uniformly globally practically asymptotically stable.

Proof 1 Considering the Lyapunov function candidate

$$
V_{v}\left(\mathbf{z}, \mathbf{e}_{v}\right)=\frac{1}{2} \mathbf{e}_{v}^{T} \mathbf{e}_{v}+\frac{1}{2} \mathbf{z}^{T} \mathbf{L}_{2} \mathbf{z}+\frac{1}{2} \mathbf{z}^{T} \mathbf{e}_{v}
$$

which is positive definite for $\mathbf{L}_{2, m}>1 / 4$, and where $\mathbf{L}_{i, m}$ (resp. $\quad \mathbf{L}_{i, M}$ ) designates the minimum (resp. maximum) eigenvalue of $\mathbf{L}_{i}$. Differentiating along the closed-loop trajectories it follows that for any positive constant $\lambda$

$$
\begin{aligned}
\dot{V}_{v}\left(\mathbf{z}, \mathbf{e}_{v}\right) \leq & -\left(\mathbf{L}_{1, m}-\frac{1}{2}-\frac{\lambda}{4} \mathbf{L}_{1, M}-\frac{3 V_{M}}{2\left\|\left(\mathbf{e}_{v}, \mathbf{z}\right)\right\|}\right)\left\|\mathbf{e}_{v}\right\|^{2} \\
& -\frac{1}{2}\left(\mathbf{L}_{2, m}-\frac{1}{2 \lambda} \mathbf{L}_{1, M}-\frac{3 V_{M}}{\left\|\left(\mathbf{e}_{v}, \mathbf{z}\right)\right\|}\right)\|\mathbf{z}\|^{2}
\end{aligned}
$$

since $\|\mathbf{J}(\mathbf{x})\| \leq 1$. A proper choice of gain matrices $\mathbf{L}_{1}$ and $\mathbf{L}_{2}$ generate the following bound

$$
\left\|\mathbf{e}_{v}\right\|^{2}+\|\mathbf{z}\|^{2} \geq \delta_{v}^{2} \quad \Rightarrow \quad \dot{V}_{v}\left(\mathbf{z}, \mathbf{e}_{v}\right) \leq-\left\|\mathbf{e}_{v}\right\|^{2}-\|\mathbf{z}\|^{2}
$$

where $V_{v}$ is positive definite and radially unbounded, and the error dynamics are uniformly practically asymptotically stable by the definition in Chaillet (2006), which implies that the region to which the solutions converge - from any initial condition - can be reduced as much as desired by enlarging $\mathbf{L}_{1, m}$ and $\mathbf{L}_{2, m}$.

Details of the proof can be found in Kyrkjeb $\varnothing$ et al. (2006a). 
The velocity information from the virtual system design can be utilized in the design of a coordination controller for the follower to synchronize its motion to the virtual system, and thus to the leader. Note that the body-fixed velocity $v_{v}$ is now known through the definition of the control law (11), and the velocity $\dot{\mathbf{x}}_{v}$ of the virtual system can be obtained through the kinematic relationship (8). Furthermore, due to the virtual system controller design, an expression for the acceleration of the virtual system will be partially available for control purposes. The variables available from the virtual system design to the coordination controller are

$$
\begin{aligned}
& \dot{\mathbf{x}}_{v}=\mathbf{J}\left(\mathbf{x}_{v}\right) v_{v}=-\mathbf{L}_{1} \mathbf{e}_{v}-\mathbf{L}_{2} \mathbf{z} \\
& \ddot{\mathbf{x}}_{v}=-\mathbf{L}_{1} \dot{\mathbf{e}}_{v}-\mathbf{L}_{2} \mathbf{e}_{v}=\left(\mathbf{L}_{1}^{2}-\mathbf{L}_{2}\right) \mathbf{e}_{v}+\mathbf{L}_{1} \mathbf{L}_{2} \mathbf{z}+\mathbf{L}_{1} \mathbf{J}\left(\mathbf{x}_{m}\right) v_{m}
\end{aligned}
$$

Defining the coordination errors and derivatives as $\mathbf{e}=$ $\mathbf{x}-\mathbf{x}_{v}$, the sliding surface from Slotine and Li (1987) can be used as a passive filtering of the virtual system states to design a virtual reference trajectory as

$$
\dot{\mathbf{y}}_{v}=\dot{\mathbf{x}}_{v}-\Lambda \mathbf{e} \quad \ddot{\mathbf{y}}_{v}=\ddot{\mathbf{x}}_{v}-\Lambda \dot{\mathbf{e}}
$$

where $\boldsymbol{\Lambda}>0$ is a positive definite design matrix. Through the definition of a measure of tracking

$$
\mathbf{s}=\dot{\mathbf{x}}-\dot{\mathbf{y}}_{v}=\dot{\mathbf{e}}+\Lambda \mathbf{e}
$$

an internal feedback loop is introduced where the integral term $\int_{0}^{t} \mathbf{e} d t$ is not used explicitly in the controller, but gives additional design freedom in $\boldsymbol{\Lambda}$ that simplifies the stability analysis. Denoting

$$
\ddot{\mathbf{y}}_{v}^{\prime}=\left(\mathbf{L}_{1}^{2}-\mathbf{L}_{2}\right) \mathbf{e}_{v}+\mathbf{L}_{1} \mathbf{L}_{2} \mathbf{z}-\mathbf{\Lambda} \dot{\mathbf{e}}
$$

the term $\ddot{\mathbf{y}}_{v}^{\prime}$ is available for control design. To coordinate the motion of the follower to the virtual system, the coordination control law inspired by Paden and Panja (1988) is introduced for the follower system

$\tau=\mathbf{M}(\mathbf{x}) \ddot{\mathbf{y}}_{v}^{\prime}+\mathbf{C}(\mathbf{x}, \dot{\mathbf{x}}) \dot{\mathbf{y}}_{v}+\mathbf{D}(\mathbf{x}, \dot{\mathbf{x}}) \dot{\mathbf{y}}_{v}+\mathbf{g}(\mathbf{x})-\mathbf{K}_{d} \mathbf{s}-\mathbf{K}_{p} \mathbf{e}$

where $\mathbf{K}_{p}$ and $\mathbf{K}_{d}$ are symmetric positive gain matrices give the closed-loop errors

$$
\begin{aligned}
\mathbf{M}(\mathbf{x}) \dot{\mathbf{s}}+\mathbf{C}(\mathbf{x}, \dot{\mathbf{x}}) \dot{\mathbf{s}}+\mathbf{D}(\mathbf{x}, \dot{\mathbf{x}}) \dot{\mathbf{s}}+\mathbf{K}_{d} \mathbf{S}+ & \mathbf{K}_{p} \mathbf{e}= \\
- & \mathbf{M}(\mathbf{x}) \mathbf{L}_{1} \mathbf{J}\left(\mathbf{x}_{m}\right) v_{m}
\end{aligned}
$$

Theorem 2 The closed-loop error dynamics (21) of system (3) with the control law (20) are uniformly globally practically asymptotically stable.

Proof 2 Consider the following Lyapunov function candidate

$$
V_{e}(\mathbf{e}, \mathbf{s})=\frac{1}{2} \mathbf{s}^{T} \mathbf{M}(\mathbf{x}) \mathbf{s}+\frac{1}{2} \mathbf{e}^{T} \mathbf{K}_{p} \mathbf{e}
$$

Differentiating along the closed-loop trajectories, and let $\delta_{e}$ be any given positive constant, it holds that for all $\|\mathbf{e}\|^{2}+$ $\|\mathbf{s}\|^{2} \geq \delta_{e}^{2}$

$$
\begin{aligned}
\dot{V}_{e}(\mathbf{e}, \mathbf{s}) \leq & -\left[\mathbf{D}_{m}+\mathbf{K}_{d, m}-\frac{1}{2 \delta_{e}} \mathbf{M}_{M} \mathbf{L}_{1, M}\right]\|\mathbf{s}\|^{2} \\
& -\left[\boldsymbol{\Lambda}_{m} \mathbf{K}_{p, m}-\frac{1}{2 \delta_{e}} \mathbf{M}_{M} \mathbf{L}_{1, M}\right]\|\mathbf{e}\|^{2}
\end{aligned}
$$

Proceeding as in the proof of Theorem $1, \mathbf{K}_{d, m}$ and $\mathbf{K}_{p, m}$ can be made as affine functions of $1 / \delta_{e}$ to conclude uniform global practical asymptotic stability.

Details of the proof can be found in Kyrkjebø et al. (2006a).

The control law coordinates the follower to the virtual system based on a computed virtual reference velocity from the virtual controller. The virtual system is in turn stabilized to the leader. Thus, the stability of the overall system from the follower to the leader must be analysed to conclude any stability properties for the coordination control scheme.

Theorem 3 Considering the system model (3) with the virtual system control law (11) and the coordination controller (20), the overall closed-loop system is uniformly globally practically asymptotically stable.

Proof 3 Take as a positive definite Lyapunov function candidate

$$
V(\widetilde{\eta})=\frac{1}{2} \widetilde{\eta}^{T} \mathbf{P} \widetilde{\eta}
$$

with the closed-loop errors

$$
\widetilde{\eta}=\left[\begin{array}{llll}
\mathbf{e}^{T} & \mathbf{s}^{T} & \mathbf{z}^{T} & \mathbf{e}_{v}^{T}
\end{array}\right]^{T}
$$

where

$$
\mathbf{P}=\left[\begin{array}{cccc}
\mathbf{K}_{p} & 0 & 0 & 0 \\
0 & \mathbf{M}(\mathbf{x}) & 0 & 0 \\
0 & 0 & \mathbf{L}_{2} & \frac{1}{2} \mathbf{I} \\
0 & 0 & \frac{1}{2} \mathbf{I} & \mathbf{I}
\end{array}\right]
$$

is a composition of the Lyapunov functions (13) and (22). Differentiating along the closed-loop trajectories gives

$$
\dot{V}(\widetilde{\eta})=-\widetilde{\eta}^{T} \mathbf{Q} \widetilde{\eta}+\beta\left(\mathbf{s}, \mathbf{e}_{v}, \mathbf{z}, v_{m}\right)
$$

where

$$
\mathbf{Q}=\left[\begin{array}{cccc}
\boldsymbol{\Lambda}^{T} \mathbf{K}_{p} & 0 & 0 & 0 \\
0 & \mathbf{D}(\mathbf{x}, \dot{\mathbf{x}})+\mathbf{K}_{d} & 0 & 0 \\
0 & 0 & \frac{1}{2} \mathbf{L}_{2} & \frac{1}{4} \mathbf{L}_{1} \\
0 & 0 & \frac{1}{4} \mathbf{L}_{1} & \mathbf{L}_{1}-\frac{1}{2} \mathbf{I}
\end{array}\right]
$$


and

$$
\begin{aligned}
\beta\left(\mathbf{s}, \mathbf{e}_{v}, \mathbf{z}, v_{m}\right)= & -\mathbf{s}^{T} \mathbf{M}(\mathbf{x}) \mathbf{L}_{1} \mathbf{J}\left(\mathbf{x}_{m}\right) v_{m} \\
& -\frac{1}{2} \mathbf{z}^{T} \mathbf{J}\left(\mathbf{x}_{m}\right) v_{m}-\mathbf{e}_{v}^{T} \mathbf{J}\left(\mathbf{x}_{m}\right) v_{m}
\end{aligned}
$$

Let $\delta$ be any given positive constant such that

$$
\begin{aligned}
\|\widetilde{\eta}\| \geq \delta & \Rightarrow \\
\left\|\beta\left(\mathbf{s}, \mathbf{e}_{v}, \mathbf{z}, v_{m}\right)\right\| \leq & \frac{V_{M}}{\delta}\left(\mathbf{M}_{M} \mathbf{L}_{1, M}\|\mathbf{s}\|^{2}+\frac{\|\mathbf{z}\|^{2}}{2}+\frac{\left\|\mathbf{e}_{v}\right\|^{2}}{2}\right)
\end{aligned}
$$

Consequently, in view of the proofs of Theorem 1 and 2, and repeating a similar reasoning as for (14) and (23) while choosing the minimum eigenvalue of the gain matrices $\mathbf{K}_{p}$, $\mathbf{K}_{d}, \mathbf{L}_{1}$ and $\mathbf{L}_{2}$ large enough, it holds that

$$
\dot{V}(\widetilde{\eta}) \leq-\|\widetilde{\eta}\|^{2}, \quad \forall\|\widetilde{\eta}\| \geq \delta .
$$

Since the dependency on the bound on $\beta$ (and on the gain matrices) in $1 / \delta$ is again affine the closed-loop system is uniform global practical asymptotic stability.

Details of the proof can be found in Kyrkjebø et al. (2006a).

\subsection{A dynamic observer approach}

The dynamic model-based observer approach (Kyrkjeb $\varnothing$ and Pettersen (2007)) is based on designing an error observer for the evolution of the coordination error, and then reconstructing the leader states based on these coordination errors and the states of the follower. Based on the position of the follower $\mathbf{x}$ and the position of the leader $\mathbf{x}_{m}$, a position coordination error $\mathbf{e}$ forms the basis for the coordination controller and for the dynamic model-based error observer estimating $\dot{\mathbf{e}}$ and $\ddot{\mathbf{e}}$. However, as the parameters of the mathematical model of the leader are unknown, an error observer is instead constructed using the dynamic model of the follower (which is known) together with the coordination control law - where the coordination control law is part of the correction term in the observer - as depicted in Figure 1.

Based on the estimated errors $\mathbf{e}$ and $\dot{\mathbf{e}}$ and the states of the follower $\mathbf{x}$ and $\dot{\mathbf{x}}$, the states of the leader can then be found algebraically through the definition of the coordination errors. The coordination errors and derivatives are defined as $\mathbf{e}=\mathbf{x}-\mathbf{x}_{m}$, and apply the passive filtering of (17) and the measure of tracking as defined in (18). The coordination control law is inspired by Paden and Panja (1988)

$$
\tau=\mathbf{M}(\mathbf{x}) \widehat{\ddot{\mathbf{y}}}+\mathbf{C}(\mathbf{x}, \dot{\mathbf{x}}) \widehat{\dot{\mathbf{y}}}+\mathbf{D}(\mathbf{x}, \dot{\mathbf{x}}) \widehat{\dot{\mathbf{y}}}+\mathbf{g}(\mathbf{x})-\mathbf{K}_{d} \widehat{\mathbf{s}}-\mathbf{K}_{p} \mathbf{e}
$$

to coordinate the states of the follower to the states of the leader. A full-state non-linear model-based Luenberger observer (Luenberger (1971)) is designed to estimate $\widehat{\mathbf{e}}$ and $\widehat{\mathbf{s}}$

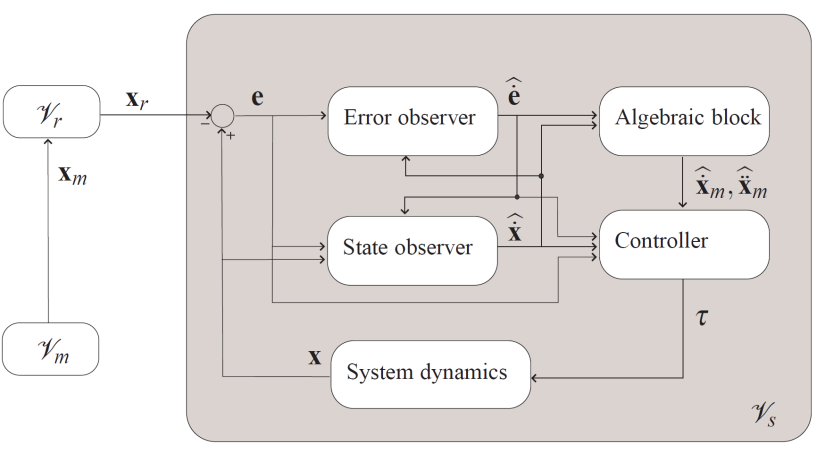

Figure 1: Schematic of the dynamic observer-controller coordination control system of the follower $\mathscr{V}_{S}$.

as

$\frac{d}{d t} \widehat{\mathbf{e}}=\widehat{\mathbf{s}}-\boldsymbol{\Lambda} \widehat{\mathbf{e}}+\mathbf{L}_{1} \widetilde{\mathbf{e}}$

$\frac{d}{d t} \widehat{\mathbf{s}}=-\mathbf{M}^{-1}(\mathbf{x})\left[\mathbf{C}(\mathbf{x}, \dot{\mathbf{x}}) \widehat{\mathbf{s}}+\mathbf{D}(\mathbf{x}, \dot{\mathbf{x}}) \widehat{\mathbf{s}}+\mathbf{K}_{d} \widehat{\mathbf{s}}+\mathbf{K}_{p} \widehat{\mathbf{e}}\right]+\mathbf{L}_{2} \widetilde{\mathbf{e}}$

where $\widetilde{\mathbf{e}}=\mathbf{e}-\widehat{\mathbf{e}}$. The closed-loop error dynamics of the system (3) and the controller (32) are

$$
\begin{array}{r}
\mathbf{M}(\mathbf{x}) \dot{\mathbf{s}}+\mathbf{C}(\mathbf{x}, \dot{\mathbf{x}}) \mathbf{s}+\mathbf{D}(\mathbf{x}, \dot{\mathbf{x}}) \mathbf{s}+\mathbf{K}_{d} \mathbf{s}+\mathbf{K}_{p} \mathbf{e}= \\
\mathbf{C}(\mathbf{x}, \dot{\mathbf{x}}) \widetilde{\mathbf{s}}+\mathbf{D}(\mathbf{x}, \dot{\mathbf{x}}) \widetilde{\mathbf{s}}+\mathbf{K}_{d} \widetilde{\mathbf{s}}+\mathbf{M}(\mathbf{x}) \widetilde{\mathbf{s}}
\end{array}
$$

where $\widetilde{\mathbf{s}}=\mathbf{s}-\widehat{\mathbf{s}}$. The estimation error dynamics are now

$$
\dot{\widetilde{\mathbf{e}}}=\widetilde{\mathbf{s}}-\left(\boldsymbol{\Lambda}+\mathbf{L}_{1}\right) \widetilde{\mathbf{e}}, \quad \dot{\widetilde{\mathbf{s}}}=\widetilde{\mathbf{\mathbf { s }}}-\left(\mathbf{M}^{-1}(\mathbf{x}) \mathbf{K}_{p}+\mathbf{L}_{2}\right) \widetilde{\mathbf{e}}
$$

Note from (35) that $\dot{\widetilde{\mathbf{s}}} \neq \widetilde{\mathbf{\mathbf { s }}}$ through the definition of the error observer in (33). Assuming for simplicity that the gain matrices $\mathbf{K}_{p}, \mathbf{K}_{d}$ and $\mathbf{L}_{1}, \mathbf{L}_{2}$ are symmetric and positive definite, a change of coordinates

$$
\overline{\mathbf{e}}=\mathbf{e}-\widetilde{\mathbf{e}}, \quad \overline{\mathbf{s}}=\mathbf{s}-\widetilde{\mathbf{s}}
$$

gives the closed-loop error dynamics of (34) by using (35) as

$$
\mathbf{M}(\mathbf{x}) \dot{\overline{\mathbf{s}}}+\mathbf{C}(\mathbf{x}, \dot{\mathbf{x}}) \overline{\mathbf{s}}+\mathbf{D}(\mathbf{x}, \dot{\mathbf{x}}) \overline{\mathbf{s}}+\mathbf{K}_{d} \overline{\mathbf{s}}+\mathbf{K}_{p} \overline{\mathbf{e}}=\mathbf{M}(\mathbf{x}) \mathbf{L}_{2} \widetilde{\mathbf{e}}
$$

The estimates of the leader states can now be found through

$$
\widehat{\dot{\mathbf{y}}}=\dot{\mathbf{x}}-\widehat{\mathbf{s}} \quad \widehat{\ddot{\mathbf{y}}}=-\boldsymbol{\Lambda}\left(\widehat{\mathbf{s}}-\boldsymbol{\Lambda} \widehat{\mathbf{e}}+\mathbf{L}_{1} \widetilde{\mathbf{e}}\right)
$$

The objective of the control law of the follower is to coordinate the follower system to the leader system based on the estimates of the leader states. The leader states are indirectly estimated through the error observer of (33) that uses the dynamic model of the follower and its control input as parameters.

Theorem 4 Considering the model (3) with the controller 
(32) and the observer (33), the closed-loop errors

$$
\widetilde{\eta}=\left[\begin{array}{llll}
\dot{\mathbf{e}}^{T} & \mathbf{e}^{T} & \widetilde{\mathbf{e}}^{T} & \widetilde{\mathbf{e}}^{T}
\end{array}\right]^{T}
$$

are globally uniformly ultimately bounded. The bound is a function of the leader acceleration $\ddot{\mathbf{x}}_{m}$.

Proof 4 Consider the Lyapunov function candidate

$$
\begin{aligned}
& V(\overline{\mathbf{s}}, \overline{\mathbf{e}}, \widetilde{\mathbf{s}}, \widetilde{\mathbf{e}})= \\
& \frac{1}{2} \overline{\mathbf{s}}^{T} \mathbf{M}(\mathbf{x}) \overline{\mathbf{s}}+\overline{\mathbf{e}}^{T} \mathbf{K}_{p} \overline{\mathbf{e}}+\frac{1}{2} \widetilde{\mathbf{S}}^{T} \mathbf{P}_{1} \widetilde{\mathbf{s}}+\frac{1}{2} \widetilde{\mathbf{e}}^{T} \mathbf{L}_{2} \widetilde{\mathbf{e}}-\widetilde{\mathbf{e}}^{T} \mathbf{P}_{2} \widetilde{\mathbf{s}}
\end{aligned}
$$

where $\mathbf{P}_{1}$ and $\mathbf{P}_{2}$ are positive definite constant matrices to be defined and (40) is positive definite through Property $P 1$ when

$$
\mathbf{P}_{1, m} \mathbf{L}_{2, m}>\mathbf{P}_{2, M}^{2}
$$

Defining the shorthand $\mathbf{L}_{3}:=\mathbf{\Lambda}+\mathbf{L}_{1}$, and introducing a constant parameter $\varepsilon>1$ used as a tuning parameter in the stability proof, the derivative of (40) along the closed-loop trajectories becomes

$$
\begin{aligned}
& \dot{V}(\overline{\mathbf{s}}, \overline{\mathbf{e}}, \widetilde{\mathbf{s}}, \widetilde{\mathbf{e}})=\frac{1}{2}\left[\begin{array}{c}
\widetilde{\mathbf{s}} \\
\widetilde{\mathbf{e}}
\end{array}\right]^{T} \mathbf{Q}_{1, \beta}\left[\begin{array}{c}
\widetilde{\mathbf{s}} \\
\widetilde{\mathbf{e}}
\end{array}\right] \\
&-\frac{1}{2}\left[\begin{array}{c}
\overline{\mathbf{s}} \\
\overline{\mathbf{e}}
\end{array}\right]^{T} \overbrace{\left[\begin{array}{cc}
\mathbf{D}(\mathbf{x}, \dot{\mathbf{x}})+\mathbf{K}_{d} & 0 \\
0 & \mathbf{\Lambda}^{T} \mathbf{K}_{p}
\end{array}\right]}^{\mathbf{Q}_{2}}\left[\begin{array}{c}
\overline{\mathbf{s}} \\
\overline{\mathbf{e}}
\end{array}\right] \\
&- \frac{1}{2}\left[\begin{array}{c}
\overline{\mathbf{s}} \\
\widetilde{\mathbf{e}}
\end{array}\right]^{T} \overbrace{\left[\begin{array}{cc}
\mathbf{D}(\mathbf{x}, \dot{\mathbf{x}})+\mathbf{K}_{d} & -\mathbf{M}(\mathbf{x}) \mathbf{L}_{2} \\
-\mathbf{M}(\mathbf{x}) \mathbf{L}_{2} & \frac{\varepsilon-1}{\varepsilon} \mathbf{L}_{2} \mathbf{L}_{3}
\end{array}\right]}^{\mathbf{Q}_{3}}\left[\begin{array}{c}
\overline{\mathbf{s}} \\
\widetilde{\mathbf{e}}
\end{array}\right] \\
&-\frac{1}{2}\left[\begin{array}{c}
\overline{\mathbf{e}} \\
\widetilde{\mathbf{e}}
\end{array}\right]^{T} \overbrace{\left[\begin{array}{cc}
\mathbf{\Lambda}^{T} \mathbf{K}_{p} & -\mathbf{L}_{1}^{T} \mathbf{K}_{p} \\
-\mathbf{L}_{1}^{T} \mathbf{K}_{p} & \frac{\varepsilon-1}{\varepsilon} \mathbf{L}_{2} \mathbf{L}_{3}
\end{array}\right]}^{\left[\begin{array}{c}
\overline{\mathbf{e}} \\
\widetilde{\mathbf{e}}
\end{array}\right]+\beta_{N}(\cdot)}
\end{aligned}
$$

where

$\mathbf{Q}_{1, \beta}=\left[\begin{array}{cc}2 \mathbf{L}_{1} & \left(\mathbf{M}^{-1}(\mathbf{x}) \mathbf{K}_{p}-\mathbf{L}_{3}^{T} \mathbf{L}_{1}\right) \\ \left(\mathbf{M}^{-1}(\mathbf{x}) \mathbf{K}_{p}-\mathbf{L}_{3}^{T} \mathbf{L}_{1}\right) & \frac{2}{\varepsilon} \mathbf{L}_{2} \mathbf{L}_{3}-\mathbf{L}_{1}\left(\mathbf{M}^{-1}(\mathbf{x}) \mathbf{K}_{p}+\mathbf{L}_{2}\right)\end{array}\right]$

The term $\mathbf{P}_{1}=\mathbf{I}$ for simplicity, and a tuning gain on the perturbation is introduced through the choice of $\mathbf{P}_{2}=\mathbf{L}_{1}$. The perturbation term $\beta_{N}(\cdot)$ is now given as

$$
\beta_{N}(\cdot)=-\left(\widetilde{\mathbf{s}}^{T}-\widetilde{\mathbf{e}}^{T} \mathbf{L}_{1}\right) \ddot{\mathbf{x}}_{m}
$$

The term $\mathbf{Q}_{1, \beta}$ is positive definite when

$$
\mathbf{Q}_{1, \beta}>0 \Leftrightarrow \frac{\frac{4}{\varepsilon} \mathbf{L}_{2, m} \mathbf{L}_{3, m}-\mathbf{L}_{1, M}^{2}\left(\mathbf{M}_{m}^{-1} \mathbf{K}_{p, M}+\mathbf{L}_{2, M}\right)}{\left[\left(\mathbf{M}_{m}^{-1} \mathbf{K}_{p, M}-\mathbf{L}_{3, m}^{T} \mathbf{L}_{1, m}\right)\right]^{2}}>1
$$

ing the filter gain $\mathbf{\Lambda}$ of (18). The term $\mathbf{Q}_{2}$ is positive definite trivially with symmetric positive definite gains $\mathbf{\Lambda}, \mathbf{K}_{p}$ and $\mathbf{K}_{d}$, and conditions for positive definiteness for $\mathbf{Q}_{3}$ and $\mathbf{Q}_{4}$ are

$$
\begin{array}{ll}
\mathbf{Q}_{3}>0 & \Leftrightarrow \quad \frac{\frac{\varepsilon-1}{\varepsilon}\left(\mathbf{D}_{m}+\mathbf{K}_{p, m}\right) \mathbf{L}_{3, m}}{\mathbf{M}_{M}^{2} \mathbf{L}_{2, M}}>1 \\
\mathbf{Q}_{4}>0 & \Leftrightarrow \quad \frac{\frac{\varepsilon-1}{\varepsilon} \boldsymbol{\Lambda}_{m}^{T} \mathbf{L}_{2, m} \mathbf{L}_{3, m}}{\mathbf{L}_{1, M}^{2} \mathbf{K}_{p, M}}>1
\end{array}
$$

The perturbation term of (44) can thus be bounded as

$$
\beta_{N}(\cdot) \leq\left(\|\widetilde{\mathbf{s}}\|+\mathbf{L}_{1, M}\|\widetilde{\mathbf{e}}\|\right) A_{M}
$$

The closed-loop errors $\overline{\mathbf{s}}, \overline{\mathbf{e}}, \widetilde{\mathbf{s}}, \widetilde{\mathbf{e}}$, and thus $\widetilde{\eta}$ of (39), are globally uniformly ultimately bounded with the lower bound $\delta$ as

$$
\delta=\sqrt{1+\mathbf{L}_{1, M}} \sqrt{A_{M}}
$$

Details of the proof can be found in Kyrkjeb $\varnothing$ and Pettersen (2007).

Remark 1 Note that the acceleration of the leader $\ddot{\mathbf{x}}_{m}$ will be present as a non-vanishing disturbance in the observercontroller scheme, and the origin of the closed-loop error space is no longer an equilibrium. Therefore, the closedloop errors are only ultimately bounded by some function of the leader acceleration $\ddot{\mathbf{x}}_{m}$.

\subsection{Comparison of estimation principles}

In the dynamic observer approach, the error dynamics of the observer and of the coordination controller are closely interconnected due to the interplay between the error observer and the coordination (error) controller. The analysis of the stability properties of the dynamic observer scheme is involved due to the couplings between the observer and the controller, but more importantly for practical implementation this implies that the gain tuning is a complex and tedious task where observer gains influence controller performance, and vice versa (Kyrkjeb $\varnothing$ et al., 2006b).

The kinematic observer approach is based on a cascaded structure; the virtual system controller and the follower coordination controller are designed separately, and stability of each of the systems can to some extent be analyzed separately. Only the perturbation term from the design of the virtual system is carried through the dynamics of the follower, and must be ensured to be bounded to conclude stability of the overall system. The tuning of the kinematic observer approach is separated through the cascaded structure; the behaviour of the virtual system can be tuned to specify the performance of the kinematic observer, and the performance of the coordination control scheme is then tuned by utilizing information about the gains from the kinematic observer.

where the positive definiteness can be ensured through tun- 
The estimator in the kinematic observer approach is a designed kinematic system; a filter based on the kinematics of the follower, but utilizing no dynamic model information of the follower. Thus, it is possible to tune the gains of the virtual system to achieve performance that is not consistent with the system dynamics of the follower. Care should thus be taken in tuning the virtual system so that the reference trajectory for the coordination control law of the follower does not exceed any limitations of the actuators of the follower. Thus, the challenge faced when tuning the virtual system is similar to the one of designing a reference trajectory; the tracking problem must be feasible.

The error observer in the dynamic model-based observer scheme is based on the dynamic model of the follower, and thus the observer guarantees that the estimates of the states are consistent with the dynamics of the follower. The observer may thus be tuned to maximize performance, and energy considerations in regard to the actuators of the follower should be optimized through the tuning of controller gains.

Note that the dynamic observer leads to uniform ultimate boundedness of the closed-loop errors, while the kinematic observer yields practical asymptotic stability of the closed-loop errors. This means that the region of attraction in the dynamic observer scheme is linked to the size of the ultimate bound, and the ultimate bound can not be reduced independently from the size of the region of attraction. The closed-loop error dynamics of the dynamic observer scheme are thus uniformly ultimately bounded rather practically asymptotically stable.

\section{Coordination control examples}

The kinematic observer approach of Section 2.2 and the dynamic observer approach of Section 2.3 are valid for mechanical systems described by the Euler-Lagrange equations (3), and are presented here for the practical examples of synchronization control of robot manipulators, and marine vessels in underway replenishment operations.

\subsection{Robot manipulator coordination}

A robot manipulator is an Euler-Lagrange system satisfying Properties P1-P3. This paper considers $n$-degree-offreedom manipulators with revolute joints only. The robot manipulator dynamics are usually treated in two different configuration spaces: the joint space and the operational space. It will be assumed that the dimension of the operational space is equal to the dimension of the joint space $(n=r)$, and that the manipulator acts in non-singular configurations. The joint angles $\mathbf{q} \in \mathbb{R}^{r}$ and the operational space coordinates $\mathbf{x} \in \mathbb{R}^{n} \times \mathrm{SO}(3)$ constitutes two different sets of generalized coordinates for the robot manipulator, and the generalized positions in a six degree of freedom system are

$$
\begin{aligned}
& \mathbf{q}:=\left[q_{1}, q_{2}, q_{3}, q_{4}, q_{5}, q_{6}\right]^{T} \in \mathbb{R}^{6} \\
& \mathbf{x}:=[x, y, z, \phi, \theta, \psi]^{T} \in \mathbb{R}^{3} \times \operatorname{SO}(3)
\end{aligned}
$$

Note that the vector $\mathbf{x}$ of generalized coordinates has a position sub-vector $\mathbf{p}=[x, y, z]^{T} \in \mathbb{R}^{3}$, and an orientation subvector $\Theta=[\phi, \theta, \psi]^{T} \in \mathrm{SO}(3)$ (roll, pitch, yaw). The dynamic model of a robot manipulator in the joint space can be written (Sciavicco and Siciliano (1996))

$$
\mathbf{M}_{\mathbf{q}}(\mathbf{q}) \ddot{\mathbf{q}}+\mathbf{C}_{\mathbf{q}}(\mathbf{q}, \dot{\mathbf{q}}) \dot{\mathbf{q}}+\mathbf{d}_{\mathbf{q}}(\dot{\mathbf{q}})+\mathbf{g}_{\mathbf{q}}(\mathbf{q})=\tau_{\mathbf{q}}
$$

where $\mathbf{q}$ are the joint coordinates, $\mathbf{M}_{\mathbf{q}}(\mathbf{q})$ is the inertia matrix, $\mathbf{C}_{\mathbf{q}}(\mathbf{q}, \dot{\mathbf{q}})$ is the matrix of Coriolis and centripetal forces, $\mathbf{d}_{\mathbf{q}}(\dot{\mathbf{q}})$ is a general function of friction or other dissipative forces, and the gravitational forces are given in $\mathbf{g}_{\mathbf{q}}(\mathbf{x})$. The joint space model (52) belongs to the class of Euler-Lagrange systems described by (3), and the matrices satisfies Properties P1-P3.

The joint space and the operational space are related through the kinematic relationship

$$
\mathbf{x}=\mathbf{f}(\mathbf{q})
$$

computed from the geometric structure of the manipulator, and enables the computation of the end-effector position and orientation $\mathbf{x} \in \mathbb{R}^{3} \times \mathrm{SO}(3)$ based on the joint variables $\mathbf{q} \in \mathbb{R}^{6}$. The differential kinematic relationship

$$
\dot{\mathbf{x}}=\mathbf{J}(\mathbf{q}) \dot{\mathbf{q}}
$$

relates the joint space velocities $\dot{\mathbf{q}}$ to the operational space velocities $\dot{\mathbf{x}}$ (Khatib (1987)) through the Jacobian matrix $\mathbf{J}(\mathbf{q})=\frac{\partial \mathbf{f}(\mathbf{q})}{\partial \mathbf{q}}$. Through (54) the dynamic model of the manipulator can be rewritten in the operational space in the form of (3), and the matrices satisfies Properties P1-P3.

The dissipative term (4) in a robot manipulator is mainly due to viscous or static friction torques (Sciavicco and Siciliano (1996)). A viscous friction torque is given as $\mathbf{F}_{v} \dot{\mathbf{q}}$ where $\mathbf{F}_{v}$ is a diagonal matrix of viscous friction coefficients. Static friction is often simplified as $\mathbf{F}_{s} \operatorname{sgn}(\dot{\mathbf{q}})$ where $\mathbf{F}_{s}$ is a diagonal matrix and $\operatorname{sgn}(\dot{\mathbf{q}})$ is a vector of signfunctions of single joint velocities. Assuming that both effects are present in a manipulator gives the dissipative forces in the form

$$
\mathbf{d}_{\mathbf{q}}(\dot{\mathbf{q}})=\mathbf{F}_{v} \dot{\mathbf{q}}+\mathbf{F}_{s} \operatorname{sgn}(\dot{\mathbf{q}})
$$

Remark 2 Note that the friction term in (55) does not satisfy Assumption 1 due to the discontinuous nature of the sign-function. However, static or dry friction is always dissipative and can be compensated for without introducing any stability problems (cf. Paulsen and Egeland (1995)). 


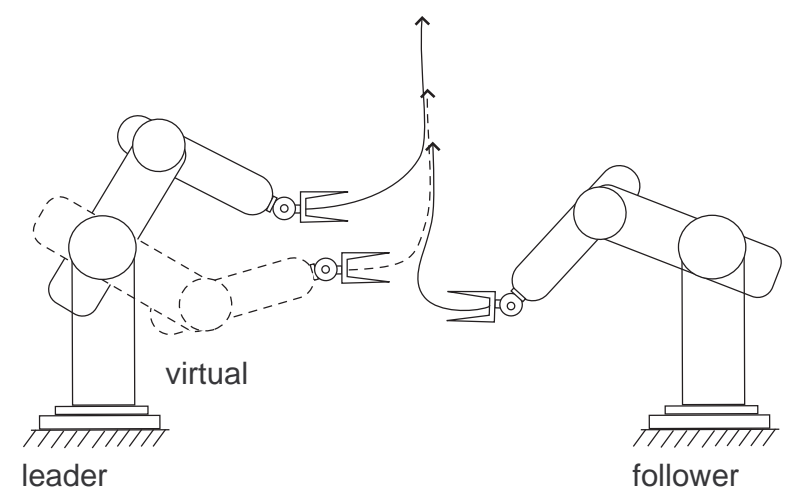

Figure 2: Leader, follower and virtual manipulator

Thus, the static friction terms can be dealt with separately or left out of the dissipative term in the stability analysis. The dissipative forces in (55) satisfy Assumption 1 when the stabilizing static friction term is ignored or dealt with separately.

Remark 3 Note also that the vector $\Theta=[\phi, \theta, \psi]^{T}$ (roll, pitch, yaw) constitutes a minimal representation of orientation, but not necessarily a singularity-free representation for which the full rotation matrix or a unit quaternion representation should be used. The manipulator may also encounter physical singularities at the boundary of its work space, or as internal singularities generally caused by the alignment of two or more axes of motion or by particular end-effector configurations (cf. Sciavicco and Siciliano (1996)) where techniques such as the damped least-squares inverse kinematic approach (cf. Chiaverini et al. (1994)) may be employed.

The robot manipulator model is now in the form of (3), and assuming that the kinematic relationship for both manipulators are known in (53), the two robot manipulators can be synchronized in the operational space using the kinematic observer approach of Section 2.2 with a virtual manipulator as shown in Figure 2, or the dynamic observer approach of Section 2.3. Note that the proposed virtual manipulator design of this section coordinates two robot manipulators in the operational space, and not in the joint space as in Rodriguez-Angeles and Nijmeijer (2001) and Bondhus et al. (2004). Hence, the coordination scheme is formulated in the task space of the robot manipulator, which is more suitable to applications where the robot manipulators should follow a geometrically specified motion, or in applications where two robots should manipulate large rigid structures. Thus, it is inherently assumed that the image of the forward kinematics of the leader must be contained within the image of the forward kinematics of the follower. Note also that if measurements of the operational space position variables $\mathbf{x}$ and $\mathbf{x}_{m}$ are available, the observer designs do not require that the direct kinematic function $\mathbf{f}(\cdot)$ is known explicitly.

No knowledge of the desired trajectory of the leader is assumed. In practice, this relates to the problem of synchronizing a follower robot manipulator to a leader robot manipulator for which only the length and type of the joints are known, and where only the joint position and orientation vector $\mathbf{q}_{m}$ is measured. Also, the restriction of knowing the velocity of the follower manipulator $\dot{\mathbf{q}}$ can be lifted by following the design in Kyrkjeb $\varnothing$ and Pettersen (2006).

\subsection{Underway replenishment}

A marine vessel is an Euler-Lagrange system, and can be written in the vectorial notation from Fossen (2002) to express the equations of motion for a marine vessel in a bodyfixed frame and an earth-fixed frame. The generalized position vector $\mathbf{x} \in \mathbb{R}^{3} \times \mathrm{SO}(3)$ and the velocity vector $v \in \mathbb{R}^{6}$ in six degrees of freedom are

$$
\begin{aligned}
& \mathbf{x}:=[x, y, z, \phi, \theta, \psi]^{T} \in \mathbb{R}^{3} \times \mathrm{SO}(3) \\
& v:=[u, v, w, p, q, r]^{T} \in \mathbb{R}^{6}
\end{aligned}
$$

Note that the vector $\mathbf{x}$ of generalized coordinates has a position sub-vector $\mathbf{p}=[x, y, z]^{T} \in \mathbb{R}^{3}$, and an orientation sub-vector $\Theta=[\phi, \theta, \psi]^{T} \in \mathrm{SO}(3)$ (roll, pitch, yaw). The body-fixed velocity vector $v$ has a linear velocity sub-vector $\mathbf{v}=[u, v, w]^{T} \in \mathbb{R}^{3}$ (surge, sway, heave), and an angular velocity sub-vector $\omega=[p, q, r]^{T} \in \mathbb{R}^{3}$. The $6 \mathrm{DOF}$ model of a marine vessel in the body-fixed reference frame can be written (Fossen (2002))

$$
\mathbf{M}_{v} \dot{v}+\mathbf{C}_{v}(v) v+\mathbf{D}_{v}(v) \dot{v}+\mathbf{g}_{v}(\mathbf{x})=\tau_{v}
$$

where $\mathbf{x}$ is the earth-fixed position and orientation vector, and $v$ is the body-fixed velocity vector. The inertia matrix $\mathbf{M}_{v}(v)$ includes added mass effects, and is positive definite and constant. The Coriolis and centripetal matrix $\mathbf{C}_{v}(v)$ is skew-symmetric $\left(\mathbf{C}_{v}(v)=-\mathbf{C}_{v}^{T}(v)\right)$, and the hydrodynamic damping matrix $\mathbf{D}_{v}(v)$ is non-symmetric and strictly positive $\left(\mathbf{D}_{v}(v)>0, \forall v \in \mathbb{R}^{6}\right)$. The gravitational/buoyancy forces are collected in $\mathbf{g}_{v}(\mathbf{x})$. Note that $v$ does not constitute a set of generalized coordinates, and therefore the model (58) does not satisfy Properties P1-P3 in general.

The Jacobian transformation matrix $\mathbf{J}(\mathbf{x})$ relates the body reference frame to the earth-fixed reference frame through

$$
\dot{\mathbf{x}}=\left[\begin{array}{c}
\dot{\mathbf{p}} \\
\dot{\Theta}
\end{array}\right]=\left[\begin{array}{cc}
\mathbf{R}_{b}^{n}(\Theta) & \mathbf{0} \\
\mathbf{0} & \mathbf{T}_{\Theta}(\Theta)
\end{array}\right]\left[\begin{array}{c}
\mathbf{v} \\
\omega
\end{array}\right]=\mathbf{J}(\mathbf{x}) v
$$

where $\mathbf{R}_{b}^{n}(\Theta)$ is the rotation matrix from the body frame to the earth frame, and $\mathbf{T}_{\Theta}(\Theta)$ is a transformation matrix. Substituting the kinematic equation (59), and its derivative into (58), the dynamic model in the earth-fixed reference 
frame can be written in the form of (3), and where the matrices satisfies Properties P1-P3.

The dissipative term (4) for a marine vessel is mainly caused by potential damping forces, skin friction, wavedrift damping and damping due to vortex shedding as defined in Fossen (2002). The contribution from potential damping terms is usually negligible compared to other dissipative terms. Linear skin friction is important in the lowfrequency motion of the vessel, and in addition there is a high-frequency non-linear (quadratic) skin friction contribution. Wave drift damping is the added resistance for surface vessels advancing in waves, and contributes heavily to damping in surge for higher sea-states due to the proportional dependency on the square of the significant wave height. Wave drift damping in sway and yaw is small relative to the viscous damping due to vortex shedding (drag). The damping terms contribute to both linear and non-linear dissipative effects, but in many cases it can be difficult to separate these effects, and it is convenient to write the hydrodynamic damping term in the body-fixed frame of the vessel as

$$
\mathbf{D}_{v}(v)=\mathbf{D}_{l}+\mathbf{D}_{n}(v)
$$

where $\mathbf{D}_{l}$ is the linear part of the damping matrix, and $\mathbf{D}_{n}(v)$ is the remaining non-linear damping effects. Note that for a rigid body moving through an ideal fluid, the hydrodynamic damping matrix of (60) will be real, nonsymmetric and strictly positive. Note also that the damping term of (4) satisfies $\mathbf{D}(\mathbf{x}, \dot{\mathbf{x}})>0 \forall \mathbf{x}, \dot{\mathbf{x}} \in \mathbb{R}^{6}$.

Non-linear dissipative terms are in practice difficult to identify beyond the contribution from quadratic damping terms for marine vessels, and in the discussion on dissipative terms in this paper, the following assumption will be made

Assumption 2 The bound on the dissipation vector in (6) satisfies for marine vessels

$$
\beta_{d}(\|\dot{\mathbf{x}}\|)=k_{D 1}+k_{D 2}\|\dot{\mathbf{x}}\|, \quad k_{D 1}, k_{D 2}>0
$$

Thus, the damping in the system is restricted to linear and quadratic damping.

Note that Assumption 2 satisfies Assumption 1 with $\beta_{d}(\cdot)$ as defined in (61). The marine vessel model for a surface ship is now in the form of (3), and two surface ships can now be synchronized in an underway replenishment operation where the objective is to transfer parts, personnel or fuel between two ships keeping a close formation using the kinematic observer from Section 2.2 or the dynamic observer from Section 2.3. No knowledge of the desired trajectory or commanded control signals for the leader ship is needed, and only position/heading measurements from the leader are available to the coordination control algorithm of the follower ship.
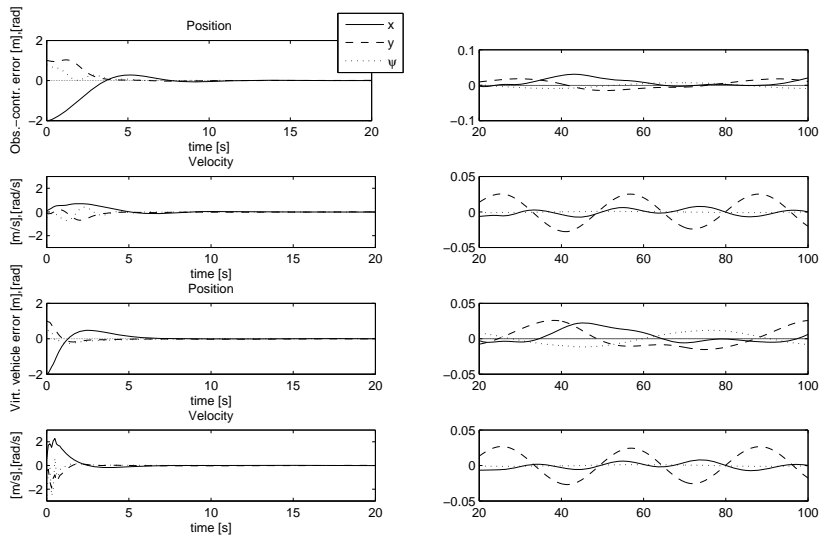

Figure 3: Nominal performance of the dynamic observer scheme (top two rows) and kinematic observer scheme (bottom two rows).

\section{Performance comparison}

To investigate the performance of the kinematic observer of Section 2.2 and the dynamic observer approach of Section 2.3, the two schemes were compared in simulations of the underway replenishment operation of Section 3.2. The practical application of underway replenishment is chosen for the performance simulations since it introduces a broader range of natural disturbances to the observer schemes than the robot synchronization application, and the two schemes are compared in terms of robustness towards model parameter errors, velocity disturbances, force disturbances, measurement noise and actuator limitations.

The leader ship in the simulation study tracks a sine wave reference trajectory $y=\sin (\varpi t)$ with frequency $\varpi=1 / 10$ $\mathrm{rad} / \mathrm{s}$ in an earth-fixed reference frame. The heading angle $\psi_{d}$ is chosen along the tangent line. The simulation model of the underway replenishment operation is based on the Froude scaled (1:70) model supply vessel Cybership II with length $1.3 \mathrm{~m}$ and weight $24 \mathrm{~kg}$ from Skjetne et al. (2004), and the nominal performance of the system without disturbances or model errors can be seen in Figure 3. More details on the simulation model can be found in Kyrkjeb $\varnothing$ (2007).

A quantitative comparison between two different observer schemes is difficult due to the fact that choosing optimal gains for both schemes requires a clearly specified optimality criterion that depends heavily on the application. Initial values are chosen to be equal for both schemes, and gains are tuned such that the schemes converge to a bounded region about the origin of approximately the same size to investigate the effect of practical disturbances rather than optimal performance. Disturbances and limitations to a specific application are particular in nature, and an impact analysis for each application should be carried out when implementing the coordination control schemes described in this paper. However, disturbances and limitations enter 

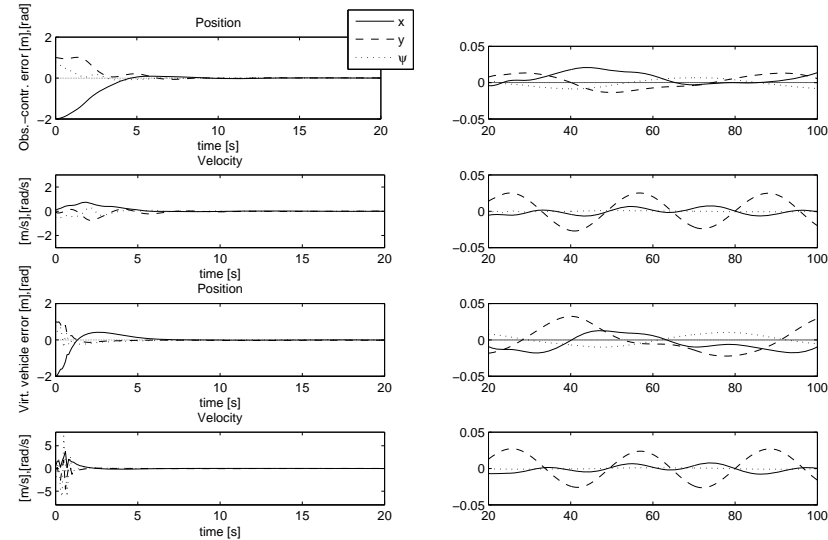

Figure 4: Performance of the dynamic observer scheme (top two rows) and kinematic observer scheme (bottom two rows) under model parameter errors.

the mathematical model of an Euler-Lagrange system (3) in similar ways for many different applications, and some general comments regarding the sensitivity of the proposed motion coordination schemes to external disturbances and actuator limitations can be made. Note that for the comparison, the simulation model is depicted as the implementation of the physical system, and the control model is the "guess" of the model utilized in the coordination controller and the observers.

\subsection{Model parameter errors}

To investigate the robustness of the observer schemes to errors in the model parameters, the parameters of the control model of the follower ship in $\mathbf{M}(\mathbf{x})$ and $\mathbf{C}(\mathbf{x}, \dot{\mathbf{x}})$ are increased by $50 \%$, while the parameters of the simulation model are unchanged. Furthermore, to address the fact that the most uncertain term in the mathematical model of a surface vessel is the damping term, the damping term $\mathbf{D}(\mathbf{x}, \dot{\mathbf{x}})$ in the control model is restricted to linear damping and the non-linear damping term present in the simulation model are disregarded. The performance of the two proposed observer schemes in the situation of model parameter errors and a linear damping assumption for the control model is shown in Figure 4. The performance is similar to the nominal performance of the system in Figure 3 except for a small increase in the velocity errors during the transient phase for the kinematic observer scheme, which suggests that the proposed observer schemes are both robust towards model parameter errors. This is also supported by practical experiments in Kyrkjeb $\varnothing$ et al. (2006b) and Kyrkjeb $\varnothing$ (2007).

\subsection{External velocity disturbances}

To investigate the robustness of the observer schemes towards external disturbances in the velocity terms, e.g., cur-
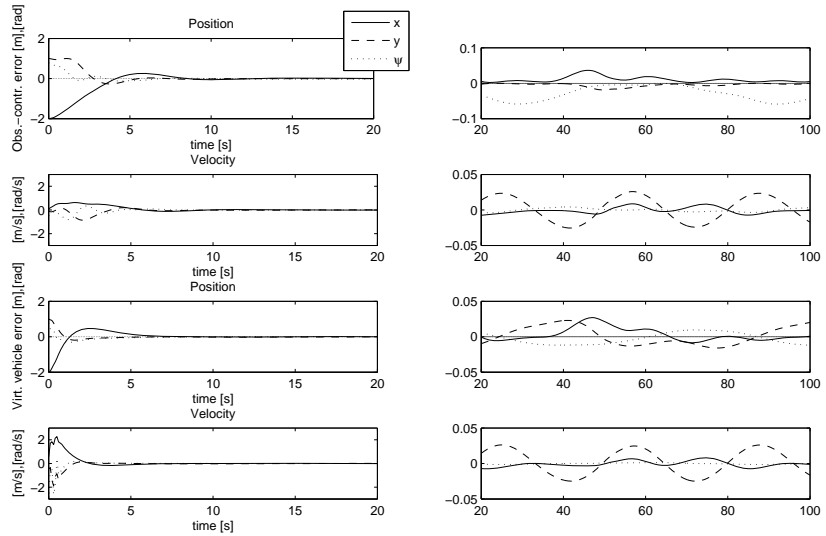

Figure 5: Performance of the dynamic observer scheme (top two rows) and kinematic observer scheme (bottom two rows) under velocity disturbances.

rents, a slowly varying ocean current $v_{c}^{n}$ is introduced from starboard in the inertial frame with the same magnitude as the nominal surge speed of the surface vessel

$$
v_{c}^{n}=-\left[\begin{array}{lll}
0 & 0.2 & 0
\end{array}\right]^{T}-\left[\begin{array}{lll}
0.1 & 0.1 & 0.1
\end{array}\right]^{T} \sin (0.1 t)
$$

in the simulation model. The control model of the follower has no knowledge of this external velocity disturbance. The external disturbance from ocean currents is chosen to be large to emphasize the effect of the velocity disturbance to clearly illustrate the impact on the coordination control schemes.

The performance of the dynamic observer scheme and the kinematic observer scheme is shown in Figure 5. The performance of the kinematic observer scheme is similar to the nominal simulations in Figure 3, while the performance of the dynamic observer scheme is slightly worse than in the nominal case. This is mainly due to the performance of the dynamic observer which is worse when the behaviour of the simulation model is different than that of the control model, and the estimates of the unknown states of the leader are less accurate. Note that the performance of the dynamic observer scheme can be improved through gain tuning to achieve similar performance as for the kinematic observer scheme.

\subsection{External force disturbances}

External force disturbances may influence the control scheme through a force vector or slowly varying bias term

$$
\mathbf{w}=\left[\begin{array}{lll}
0 & 0.1 & 0
\end{array}\right]^{T}+\left[\begin{array}{lll}
0.05 & 0.05 & 0.05
\end{array}\right]^{T} \sin (0.1 t)+\mathbf{w}_{b}
$$

with the additional zero-mean Gaussian bounded disturbance vector $\mathbf{w}_{b}$ with a standard deviation of $0.05 \mathrm{~N}$. The performance of the observer schemes is shown in Figure 

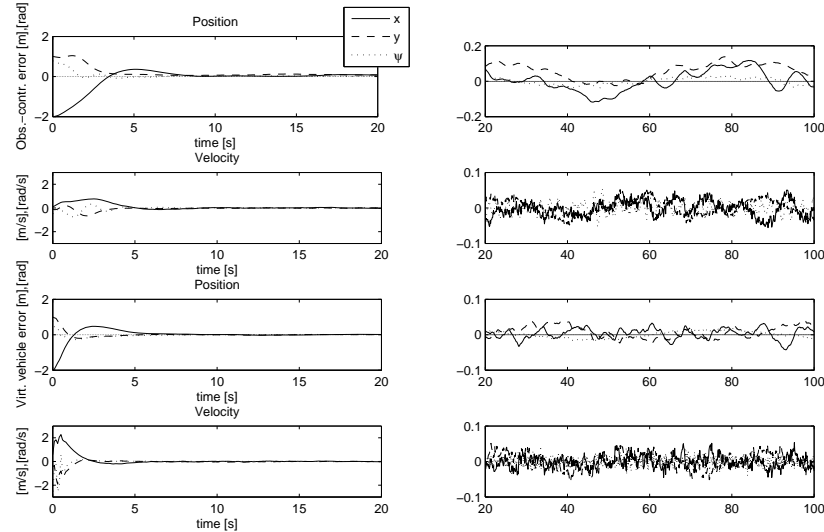

Figure 6: Performance of the dynamic observer scheme (top two rows) and kinematic observer scheme (bottom two rows) under force disturbances.

6, and the performance of the kinematic observer approach is slightly worse than in the nominal case in Figure 3, while the dynamic observer performance is more severely affected in terms of performance.

Any external force disturbances are seen by the dynamic observer coordination scheme as an unknown perturbation in acceleration, and appear in the error dynamics of the error observer (33) in the same way as the unknown leader acceleration term $\ddot{\mathbf{x}}_{m}$. Thus, unmodelled force disturbances directly influence the size of the bound on the closed-loop errors, as is clearly seen in Figure 6. Note, however, that the size of this bound can be reduced through gain tuning to yield similar performance as for the kinematic observer scheme. Note also that the definition of the virtual system control law (11) introduces an integral term in the estimation scheme, and the influence from slowly varying external disturbances are partly attenuated by the kinematic control law in the coordination control scheme.

\subsection{Measurement noise}

To investigate the effect of measurement noise and small perturbations caused by external disturbances such as wavefrequency motions generated by waves, noise and small periodic disturbances are introduced to the follower and the leader vessel through the measurement equation

$$
\mathbf{y}=\mathbf{x}+\left[\begin{array}{lll}
0.01 & 0.01 & 0.01
\end{array}\right]^{T} \sin (t)+\mathbf{v}_{y}
$$

where the zero-mean Gaussian disturbance vector $\mathbf{v}_{y}$ has a standard deviation of $0.02 \mathrm{~m}$.

The performance of the observer schemes when reducing the quality of the position measurements is shown in Figure 7 . Both the dynamic observer scheme and the kinematic observer scheme reflect the reduced quality of the measurements, and most notable are the increased velocity errors
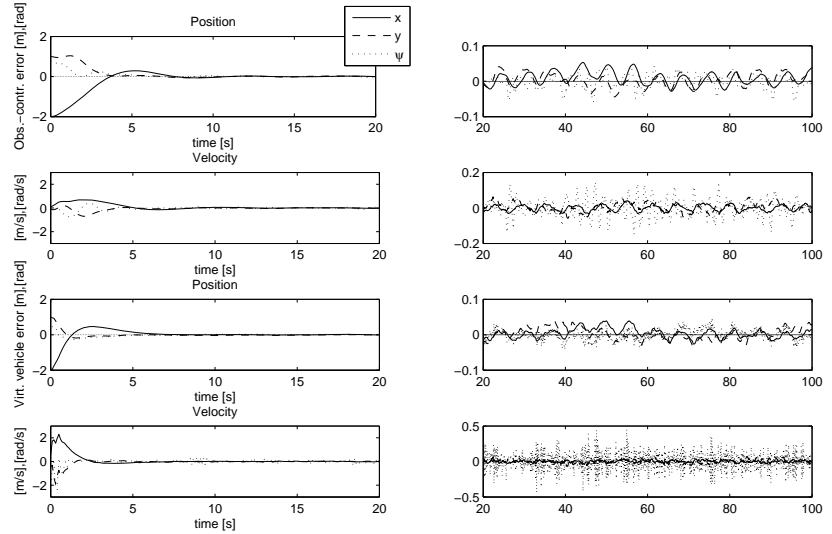

Figure 7: Performance of the dynamic observer scheme (top two rows) and kinematic observer scheme (bottom two rows) under the influence of periodic wave-frequency motions and measurement noise.

in the kinematic scheme. These errors are due to the estimation principle of the virtual system that does not employ any model-based filter based on the mathematical model of the follower, so there is no inherent noise filtering in the kinematic scheme.

Measurement noise directly adds to the size of the bounded region to which the coordination schemes converge. The size of this bounded region may be reduced through gain tuning or by introducing a band-limited filter (wave filter) in the control scheme. Note, however, that there is a lower limit to the size of this bounded region that will depend on the size of the measurement noise and nonvanishing perturbations present in the closed-loop system.

\subsection{Actuator limitations}

Limitations on the available control force from the actuators are one of the limiting factors in terms of transient performance, and may also be the determining factor in how robust automatic control systems are towards external disturbances. In practical applications, there must be sufficient control forces available to meet the performance demands of the operation, while at the same time reduce the impact from external disturbances. To investigate the effect of control saturations, force saturations are imposed on the available control force from the actuators of the follower surface vessel of $2 \mathrm{~N}$ in surge, $1.5 \mathrm{~N}$ in sway and $1.5 \mathrm{Nm}$ in yaw as determined by the actual model ship used for the simulations.

The performance of the coordination control scheme under actuator limitations is shown in Figure 8. Note the change in the length of the time vector plotted in the transient phase and after settling. The force saturations severely increase the length of the transient phase due to the limited 

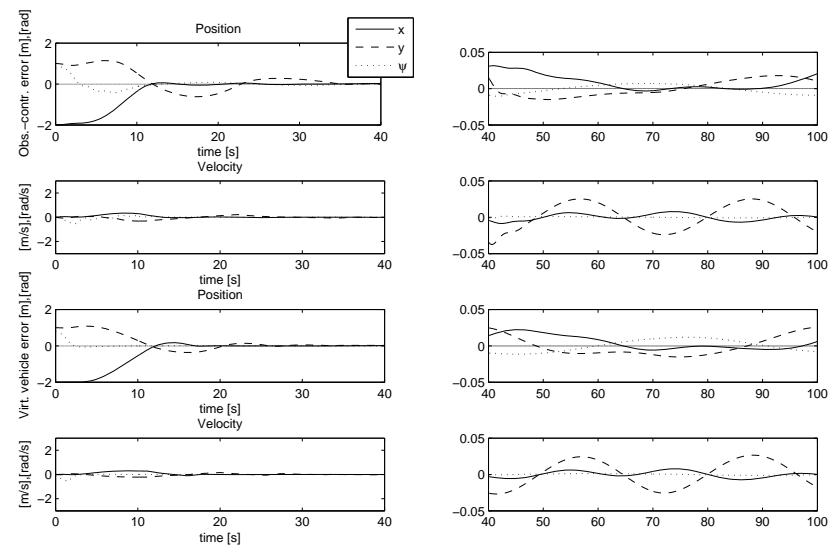

Figure 8: Performance of the dynamic observer scheme (top two rows) and kinematic observer scheme (bottom two rows) subject to actuator limitations of 2 $\mathrm{N}$ in surge, $1.5 \mathrm{~N}$ in sway and $1.5 \mathrm{Nm}$ in yaw.

amount of force available, while the performance after the transient phase is similar to the nominal performance in Figure 3.

\section{Concluding remarks}

Both the dynamic observer scheme and the kinematic observer scheme show robustness towards model parameter errors as illustrated in Figure 4. The effect of model parameter errors in the control model does not significantly influence the performance of the schemes after the initial transient phase, and the coordination schemes may be suited for practical implementation in systems with model uncertainties.

External velocity disturbances affect the performance of the dynamic observer in the dynamic observer scheme due to the unexpected behaviour of the system which is not reflected in the control model utilized in the observer. The effect of disturbances in velocity is less visible in the kinematic observer scheme since the kinematic observer does not depend on the velocity of the system. Note, however, that the external velocity disturbance introduced to the simulations shown in Figure 5 is large to emphasize the effect of the disturbance, and can be attenuated by using a different set of observer and control gains in the dynamic observer scheme. Overall, the coordination schemes show robustness towards external velocity disturbances.

External force disturbances add to the non-vanishing perturbation term in the dynamic observer scheme, and thus directly influence the size of the bounded region to which the solutions converge as seen in Figure 6. The size of this bound can be reduced by tuning the control gains, or by redesigning the control system to include an integral term or an adaptive bias estimation algorithm to counteract slowly
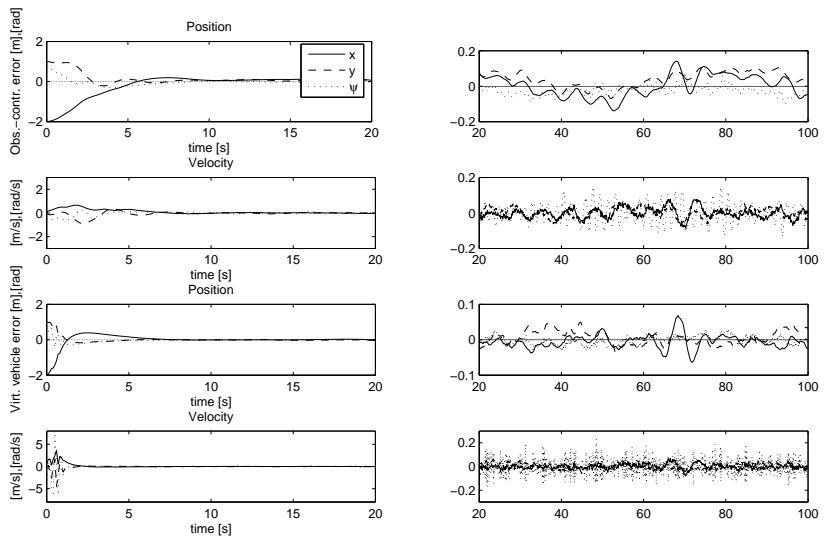

Figure 9: Performance of the dynamic observer scheme (top two rows) and kinematic observer scheme (bottom two rows) under model parameter errors, velocity disturbances, force disturbances and measurement noise.

varying disturbances appearing as external forces to the control system. The performance of the kinematic observer scheme is less affected by the introduction of a slowly varying disturbance force term due to the integral action provided through the definition of the kinematic control law.

The dynamic observer scheme utilizes a model to provide estimates of the errors used in the control scheme. Under the influence of measurement noise, the measurements are filtered through this second-order filter (the dynamic model of the follower), and thus wild-points and noisy measurements are attenuated intrinsically in the scheme. The observer is a separate model-based dynamic system that provides estimates of the unknown states of the leader whether it is updated with measurements or not, and therefore provides some robustness towards measurement noise in practical applications.

The kinematic observer scheme utilizes a virtual system control law to estimate the unknown states of the leader. Under the influence of measurement noise on the leader position measurements, there is no model-based filter inherently applied to the measurements, and therefore the scheme is more sensitive to fast transients in the measurement signals. This is also confirmed in experiments in Kyrkjeb $\varnothing$ (2007) and in the velocity errors of Figure 7. Note that this issue can be addressed by designing a separate filter to remove wild-points and band-limit the measurement signal to reduce the influence of measurement noise.

The overall performances of the coordination control schemes are shown in Figure 9, where the model parameter errors, the velocity and force disturbances and the measurement noise defined in this section are imposed on the follower simultaneously. The impact from the external disturbances influences the performance of the coordination control schemes similarly, although the dynamic observer 
scheme suffers from the increased size of the non-vanishing perturbation due to the force disturbances. Note that the performance of the dynamic observer scheme can be improved to show a level of performance similar to that of the virtual vehicle scheme by tuning the control gains.

Overall, both the dynamic observer scheme and the kinematic observer scheme are shown to exhibit robustness towards external disturbances, measurement noise and actuator limitations. The performance of both schemes is sensitive to the performance of the observer schemes, and thus care should be taken in tuning the coordination control schemes. The dynamic observer scheme is sensitive to the tuning of the observer: a choice between the convergence rate and the sensitivity towards disturbances has to be made. In the kinematic observer scheme, care should be taken in designing the virtual system velocity such that it only demands velocities and accelerations within the range of the actuators of the follower, and hence provides a reference trajectory that complies with the system dynamics of the follower.

Note that although the discussion on robustness in this section is carried out in the framework of underway replenishment for ships, the results are an indication of the robustness of the schemes in different applications within the Euler-Lagrange framework. Note also that the simulation study presented in this section does not necessarily reflect the true magnitude and nature of disturbances encountered in a full-scale operation, and should thus be regarded as a indication of the impact of external disturbances only.

\section{Acknowledgments}

The author is very grateful to Professor Kristin Y. Pettersen at the Norwegian University of Science and Technology for guidance and discussions regarding most of the work that this paper is based on, and Professor Elena Panteley, Professor Antonoio Loría and Dr. Antoine Chaillet at Laboratoire des Signaux et Systems, CNRS, France, for discussions and inputs regarding the kinematic observer scheme.

\section{References}

Al-Hiddabi, S. A. and McClamroch, N. H. Tracking and maneuver regulation control for nonlinear nonminimum phase systems: Application to flight control. IEEE Trans. on Control Systems Technology, 2002. 10(6):780 - 792. doi:10.1109/TCST.2002.804120.

Bondhus, A. K., Pettersen, K. Y., and Nijmeijer, H. Masterslave synchronization of robot manipulators. In Proc. 6th IFAC Symp. on Nonlinear Control Systems. Stuttgart, Germany, pages 591 - 596, 2004.
Chaillet, A. Staiblité et Robustesse des Cascades Nonlinéaires et Application aux Systèmes Mécaniques. Ph.D. thesis, L'Université Paris XI Orsay, Supélec, France, 2006.

Chiaverini, S., Siciliano, B., and Egeland, O. Review of the damped least-squares inverse kinematics with experiments on an industrial robot manipulator. Control Systems Technology, IEEE Transactions on, 1994. 2(2):123134. doi:10.1109/87.294335.

Choi, K., Yoo, S., Park, J., and Choi, Y. Adaptive formation control in absence of leader's velocity information. Control Theory Applications, IET, 2010. 4(4):521-528. doi:10.1049/iet-cta.2009.0074.

Crowley, J. Asynchronous control of orientation and displacement in a robot vehicle. In Proc. 1989 IEEE Int. Conf. on Robotics and Automation. Scottsdale, AZ, USA, pages 1277 - 1282, 1989. doi:10.1109/ROBOT.1989.100156.

Encarnacao, P. and Pascoal, A. Combined trajectory tracking and path following: An application to the coordinated control of autonomous marine craft. In Proc. 40th IEEE Conf. on Decision and Control. Orlando, FL, USA, pages 964 - 969, 2001. doi:10.1109/cdc.2001.980234.

Fossen, T. I. Marine Control Systems: Guidance, Navigation, and Control of Ships, Rigs and Underwater Vehicles. Marine Cybernetics, Trondheim, Norway, 2002.

Fradkov, A., Gusev, S., and Makarov, I. Robust speedgradient adaptive control algorithms for manipulators and mobile robots. In Proc. 30th IEEE Conf. on Decision and Control. Brighton, England, pages 3095 - 3096, 1991. doi:10.1109/CDC.1991.261122.

Goldstein, H., Poole, C., and Safko, J. Classical Mechanics. Pearson Education, Addison Wesley, 3rd edition, 2002.

Gusev, S., Makarov, I., Paromtchik, I., Yakubovich, V., and Laugier, C. Adaptive motion control of a noholonomic vehicle. In Proc. 1998 IEEE Int. Conf. on Robotics and Automation. pages 3285 - 3290, 1998. doi:10.1109/ROBOT.1998.680945.

Khatib, O. A unified approach for motion and force control of robot manipulators: The operational space formulation. IEEE Journal of Robotics and Automation, 1987. RA-3(1):43 - 53. doi:10.1109/JRA.1987.1087068.

Kyrkjebø, E. Motion Coordination of Mechanical Systems: Leader-Follower Synchronization of EulerLagrange Systems using Output Feedback Control. Ph.D. thesis, Norwegian University of Science and Technology, Dep. of Engineering Cybernetics, Trondheim, Norway, 2007. 
Kyrkjebø, E., Panteley, E., Chaillet, A., and Pettersen, K. Y. Group Coordination and Cooperative Control, volume 336 of Lecture Notes in Control and Information Systems, chapter A Virtual Vehicle Approach to Underway Replenishment, pages $171-189$. Springer Verlag, Tromsø, Norway, 2006a. doi:10.1007/11505532_10.

Kyrkjebø, E. and Pettersen, K. Y. Output synchronization control of Euler-Lagrange systems with nonlinear damping terms. In Proc. 44th IEEE Conf. on Decision and Control and European Control Conf. Sevilla, Spain, pages 4951 - 4957, 2005. doi:10.1109/cdc.2005.1582946.

Kyrkjebø, E. and Pettersen, K. Y. A virtual vehicle approach to output synchronization control. In Proc. 45th Conf. on Decision and Control. San Diego, USA, 2006. doi:10.1109/CDC.2006.377082.

Kyrkjebø, E. and Pettersen, K. Y. Leader-follower output reference state feedback synchronization control of eulerlagrange systems. In Proc. Mediterranean Conf. on Control and Automation. Athens, Greece, 2007.

Kyrkjebø, E. and Pettersen, K. Y. Operational space synchronization of two robot manipulators through a virtual velocity estimate. Modeling, Identification and Control, 2008. 29(2):59-66. doi:10.4173/mic.2008.2.3.

Kyrkjebø, E. and Pettersen, K. Y. A kinematic versus dynamic observer approach to coordination control in terms of estimation principle and practical performance. In $E u$ ropean Control Conference. 2009.

Kyrkjebø, E., Pettersen, K. Y., Wondergem, M., and Nijmeijer, H. Output synchronization control of ship replenishment operations: Theory and experiments. Control Engineering Practice, 2006b. 15(6):741 - 755. doi:10.1016/j.conengprac.2006.07.001.

Lefeber, A. A. J. Tracking Control of Nonlinear Mechanical Systems. Ph.D. thesis, University of Twente, The Netherlands, 2000.

Loría, A., Kelly, R., Ortega, R., and Santibanez, V. On global output feedback regulation of eulerlagrange systems with bounded inputs. IEEE Trans. on Automatic Control, 1997. 42(8):1138 - 1143. doi:10.1109/9.618243.

Luenberger, D. G. An introduction to observers. IEEE Trans. on Automatic Control, 1971. 16(6):596 - 602. doi:10.1109/TAC.1971.1099826.

Marino, R. and Tomei, P. Nonlinear Control Design: Geometric, Adaptive and Robust. Prentice Hall, 1995.
Nijmeijer, H. and Fossen, T. I. New Directions in Nonlinear Observer Design, volume 244 of Lecture Notes in Control and Information Sciences. Springer Verlag, London, 1999. doi:10.1007/BFb0109917.

Nijmeijer, H. and Mareels, I. An observer looks at synchronization. IEEE Trans. on Circuits and Systems I 44, 1997. pages 882 - 890. doi:10.1109/81.633877.

Nijmeijer, H. and Rodriguez-Angeles, A. Synchronization of Mechanical Systems, volume 46. World Scientific Series on Nonlinear Science, Series A, 2003.

Nishigami, K., Yasuda, Y., and Hirata, K. An output synchronization control design for systems with input magnitude constraints. In ICCAS-SICE, 2009. pages 30773082, 2009.

Ortega, R. and Spong, M. W. Adaptive motion control of rigid robots: A tutorial. Automatica, 1989. 25(6):877 888. doi:10.1016/0005-1098(89)90054-X.

Paden, B. and Panja, R. Globally asymptotically stable "pd +" controller for robot manipulators. Int. Journal of Control, 1988. 47(6):1697 - 1712. doi:10.1080/00207178808906130.

Paulsen, M. J. and Egeland, O. Tracking controller and velocity observer for mechanical systems with nonlinear damping terms. In Proc. 3rd European Control Conf. Rome, Italy, 1995.

Peng, Z., Wang, D., Chen, Z., Hu, X., and Lan, W. Adaptive dynamic surface control for formations of autonomous surface vehicles with uncertain dynamics. Control Systems Technology, IEEE Transactions on, 2013. 21(2):513-520. doi:10.1109/TCST.2011.2181513.

Pettersen, K. Y. and Nijmeijer, H. Tracking control of an underactuated surface vessel. In Proc. 37th IEEE Conf. on Decision and Control. Tampa, FL, USA, pages 4561 - 4566, 1998. doi:10.1109/cdc.1998.762046.

Pogromsky, Y. A. and Nijmeijer, H. Observer based robust synchronization of dynamical systems. Int. Journal on Bifurcation and Chaos, 1998. pages 2243-2254. doi:10.1142/S0218127498001832.

Rodriguez-Angeles, A. and Nijmeijer, H. Coordination of two robot manipulators based on position measurements only. Int. Journal of Control, 2001. 74:1311 - 1323. doi:10.1080/00207170110065893.

Salichs, M., Puente, E., Gachet, D., and Moreno, L. Trajectory tracking for a mobile robot - an application to contour following. In Proc. Int. Conf. on Industrial Electronics, Control and Instrumentation. Kobe, pages 1067 - 1070, 1991. doi:10.1109/iecon.1991.239143. 
Sciavicco, L. and Siciliano, B. Modeling and Control of Robot Manipulators. McGraw-Hill Series in Electrical and Computer Engineering. McGraw-Hill, 1996.

Skjetne, R., Smogeli, Ø. N., and Fossen, T. I. A nonlinear ship maneuvering model: Identification and adaptive control with experiments for a model ship. Modeling, Identification and Control, 2004. 25(1):3 - 27. doi:10.4173/mic.2004.1.1.
Slotine, J.-J. E. and Li, W. Adaptive manipulator control a case study. In Proc. 1987 IEEE Int. Conf. on Robotics and Automation. pages 1392 - 1400, 1987. doi:10.1109/ROBOT.1987.1087895.

Spong, M. W. and Vidyasagar, M. Robot Dynamics and Control. John Wiley \& Sons, New York, 1989. 